\title{
Gaining Access to Decentralised Library Resources Using Location-Aware Services
}

\author{
Bjarne Sletten Olsen and Ingeborg T. Sølvberg \\ Norwegian University of Science and Technology \\ Department of Computer and Information Science \\ Sem Sælands vei 7-9 \\ NO-7491 Trondheim \\ Norway \\ bjarnesl@stud.ntnu.no \\ http://www.idi.ntnu.no
}

\begin{abstract}
The paper describes a prototype that enables library users to use their mobile devices to find the physical location of specific services or objects in a branch of a distributed library. It guides the users to this location using external map services, location-awareness and navigational tools. The architecture of the system is briefly described together with the integrated services.
\end{abstract}

Keywords: Location-aware applications, mobile applications, library resources.

\section{Introduction}

As the number of mobile phones sold reaches new heights every day, ubiquitous computing is becoming a natural part of the modern life, and new applications emerge, providing functionalities that not long ago would have been unthinkable. Examples of this are WhosHere that enables users to broadcast their profile and to detect friends or people with common interests nearby[1], and Sekai Camera that enables users to tag real-life objects with comments using the camera on the mobile phone along with GPS[2]. Many of these functionalities take advantage of the location of the user. Such location-aware applications are able to provide contextual information, reconfigure, trigger actions or select nearby objects based on this location. In a geographical area there normally are several libraries or library branches, each holding documents on different subjects. Usually the library catalogues are available using a web-based search. The system described in this paper enables library users to use their mobile devices to find the physical location of specific services or objects in a branch of the library, and to guide the users to this location using maps and navigational tools. In this paper we describe a prototype that demonstrates the possibilities at the library of the Norwegian University of Science and Technology (NTNU). An overview of the functionality is presented along with the design of the prototype as well as plans 


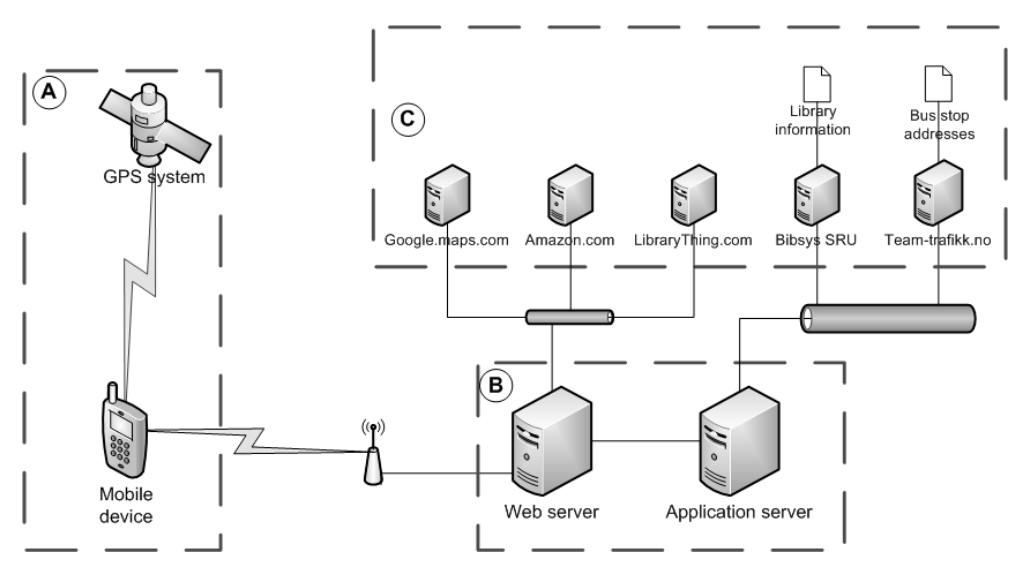

Fig. 1. Overall design with three modules. The Mobile application (A), the server-side application (B) and adapters and services (C).

for further development. The goal of this project is to demonstrate the possibilities offered by location-aware applications, as well as to show how already existing services can be integrated with this system.

\section{The Prototype}

The system consists of three modules as shown in Figure 1. Module A is the application running on the mobile device, handling communication with the GPS system. Module B is the part of the application running on servers and consists of the web server, the application server and the local database. Module $\mathrm{C}$ consists of adapters that handles communication with the external services, ensuring an identical interface for the application server, independent of what service the information is to be retrieved from. The server-side application communicates with the following services: BIBSYS grants access to the Norwegian library catalogue, containing more than 4.3 million titles, including the holdings of NTNU Library's 11 branches[3]. Google maps[4] provide a service that enables the construction of JavaScript-based maps. These constructors accept parameter's representing information that can be included in the map, such as location of Points of Interest (POI). The system is able to retrieve bus schedules from the local bus company Team Trafikk[5]. They provide a natural text search engine that responds to queries on bus schedules. The queries have to be on the form "When is the next bus for point A leaving from point B?". The response is in natural text, and has to be parsed. Amazon.com and Librarything.com are used as sources for book cover images.

\subsection{A Walk-Through Example}

A library user is in the city and needs a specific book right away. A search in BIBSYS, using the prototype running on his or hers mobile device, informs the 


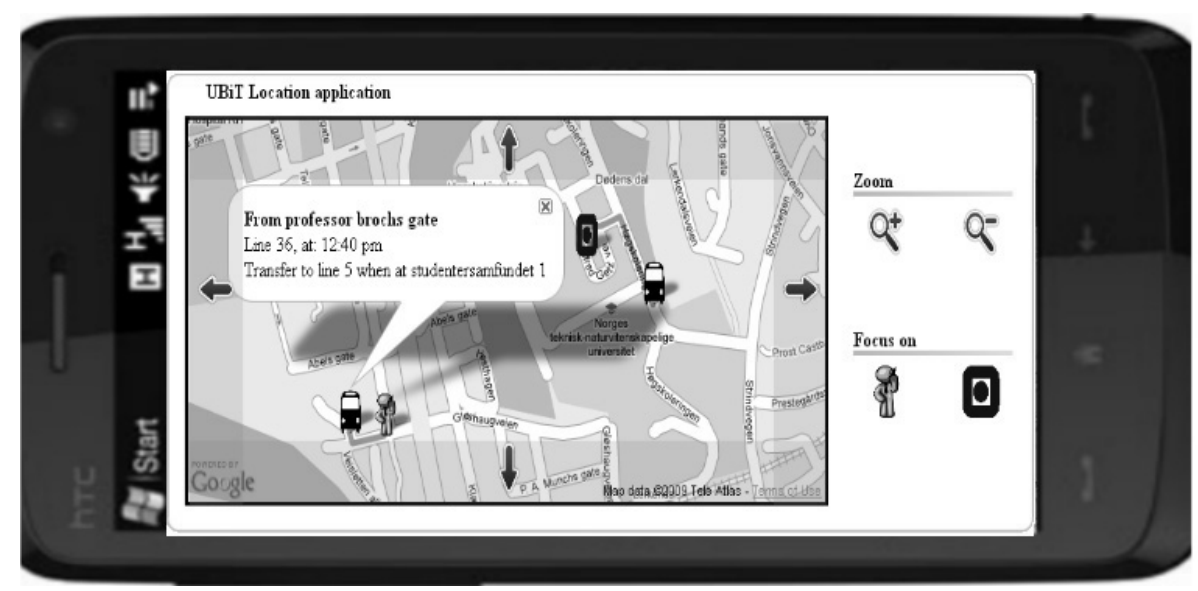

Fig. 2. Running prototype with the user, the departure bus stop, the arrival bus stop and the library indicated

user that the desired book resides in several of the university libraries branches. Information on the status of the document(chekd out, available and so on) is part of the BIBSYS system, and access to information is not provided as of now. The user selects the nearest library with the given document. The system generates a map, indicating the last registered position of the user as well as the position of the selected branch library. When the user wants to go to the branch library by bus, the system queries the local bus company on how to get there. A route to the nearest bus stop with connections to the library is then displayed on the map, along with the time of the next departure. The example is shown in Figure 2. When she or he arrives at the library, a map of the library is made available and guides the user to the shelf in which the document resides.

\section{The System and Technologies}

The mobile applicaiton was written using $\mathrm{C}^{1}$. As newer browsers are already experimenting with the direct access of user coordinates through JavaScript, a stand alone application will not be necessary in the future, and the present mobile-side application is used only for testing. As platform independence is not required for the server-side application, this is written using $\mathrm{C} \#$. One of the goals with the development of the prototype is to demonstrate that integration with other existing services is feasible. An adapter is created for each of the services the prototype needs to communicate with. In order to add new services a new adapter must be created for each service, and the map genereation must be updated to handle the new information available.

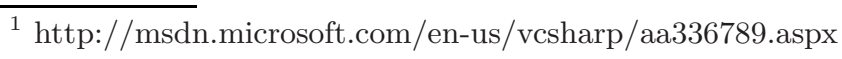




\section{Future Work}

As the system described in this paper is a prototype, there are some services that are not yet included, although planned for. One of these is the addition of a news-service, presenting the user with the latest news for the nearby area in map. This can be displayed either on city-, district- or street-level depending on the quality of the location-information associated with the news. As not all mobile devices support the use of GPS, some WiFi based location services have been considered. In the city of Trondheim, where NTNU resides, a localisation service named GeoPos[7] is available. This enables localisation services on all devices with a WiFi card installed. This would add the possibility of indoor-localisation to the application, facilitating the creation of additional services.

\section{References}

1. WhosHere, http://myrete.com/WhosHere.html

2. Gizmodo, http://www.gizmodo.com.au/2008/09/sekai_camera_turns_on_worlds_ balloon_help-2.html

3. BISBSYS Ask, http://www.bibsys.no/wps/wcm/connect/BIBSYS+Eng/

4. Google Maps API, http://code.google.com/apis/maps/

5. Team Trafikk home page, http://team-trafikk.no/

6. JSR 179: Location API for J2ME, http://www.jcp.org/en/jsr/detail?id=179

7. GeoPos, http://www.geopos.no/geopos/ 